Article

\title{
The Carabattola-Vibroacoustical Analysis and Intensity of Acoustic Radiation (IAR)
}

\author{
Lamberto Tronchin ${ }^{1, *(\mathbb{D})}$, Massimiliano Manfren ${ }^{2}$ and Vincenzo Vodola ${ }^{1}$ \\ 1 Department of Architecture, University of Bologna, Via dell'Università 50, 47521 Cesena, Italy; \\ vincenzo.vodola2@unibo.it \\ 2 Faculty of Engineering and Physical Sciences, University of Southampton, Highfields, \\ Southampton SO17 1BJ, UK; m.manfren@soton.ac.uk \\ * Correspondence: lamberto.tronchin@unibo.it
}

Received: 21 August 2019; Accepted: 15 January 2020; Published: 16 January 2020

\begin{abstract}
Among the studies of musical instruments, one important, sometime underestimated discipline, is represented by ethnomusicology. The acoustic analyses on ethnic musical instruments (M.I.) are much more infrequent if compared to those on classical M.I. This article deals with the vibro-acoustic analysis on one of the most unknown ethnic, Italian M.I., i.e., the carabattola (also called battola), which used to be played in Italy until the late 1960s during the Holy Thursday before Easter. The study includes modal analysis and Intensity of Acoustic Radiation measured on an original carabattola, which was played in the Romagna area until the early twentieth century. After a brief overview about the theory of acoustic and vibrational analysis on musical instruments, the Intensity of acoustic radiation and its correlation with modal analysis are recalled, based on previous studies. In the experimental part of the article, the measurements conducted on the carabattola are described. Afterwards, the results obtained both from modal analysis and IAR measurements are analyzed and compared with other measurements previously conducted on musical (particularly percussion) instruments and commented.
\end{abstract}

Keywords: sound efficiency; intensity of acoustic radiation (IAR); Carabattola; modal analysis

\section{Introduction}

The physics of musical instruments represents one of the most intriguing field of acoustics, especially for those scientists that are normally involved in the preservation of cultural heritage. There are different techniques to study the vibro-acoustical behavior of musical instruments; between them, modal analysis and acoustic radiation are usually used. However, some other techniques have been developed starting from these fundamental methods.

The studies on the physics and acoustics of musical instrument that were carried out in the last 30 years normally regarded classical musical instruments, especially violins [1], piano [2,3], wind instrument [4], and other musical instruments. Only a few studies have analyzed other musical instruments [5]. This was due to the request of knowledge of the sound characteristics of those instruments from lutherie, industrial manufactory companies, researchers, curators, collectors, museums, historians, musicians, theatre companies and all parties involved with preservation and restoration of those important, valuable objects. One more reason for studying the sound characteristics of musical instruments from the physical perspective is the emulation of their sound characteristics [6] by means of measurements of impulse responses [7] and convolution with dry music, including nonlinear properties $[8,9]$. 
On the other hand, other musical instruments have been developed or invented for several reasons, sometimes very different from each other, but the scientific community did not pay proper attention to them. The carabattola, sometime called battola, belongs to this category of musical instruments (M.I.).

In order to determine the acoustic characteristics of M.I., acoustic radiation (like acoustic impedance or admittance) is one of the most important physical parameters utilized to characterize their properties. Sound radiation is closely linked to modal patterns, and a connection should therefore have occurred between resonance frequencies and sound production in vibration constructions. Evidence of the negative correlation between acoustic radiation and Frequency Response Function (FRF) of membranes or plates in instruments, such as piano and harpsichord, has been identified in earlier studies by Suzuki [10] and Giordano [11]. The complexity of pianos and harpsichord was perhaps the reason for the negative correlation, and their complex structure could have impeded their understanding of their sound radiation.

Percussion instruments, on the other side, are comparatively straightforward, and frequency response studies, modal analysis, acoustic radiation and the relationship between FRF and noise radiation can be readily discovered [12]. The comparison between experimental modal patterns and previously outcomes could suggest the most appropriate measurement technique for characterizing vibro-acoustical properties of musical instruments. For tympani, where sound generation and modal analysis are related, a vibro-acoustic parameter was needed, capable of correctly correlating noise output with FRF. Applications of this extend beyond musical acoustics into the modeling of musical instruments in auditoria. The search of the link between sound radiation and modal analysis in musical instruments has been an issue for several years.

\section{The Carabattola}

In western music there are plenty of musical instruments normally played since the beginning of prehistoric ages. Most of them were idiophonic M.I., in which the sound was obtained by hitting the bore with wooden or (later) metallic elements. Moreover, there are several instruments which were invented not for generating pitched music but rather for producing acoustic effects, mainly for specific religious circumstances. Among them, in Mediterranean Europe, several ethnographic instruments have been utilized since the early middle ages almost up to the present. The carabattola is one of these M.I.

The carabattola, also known as battola, is an extremely uncommon idiophone instrument of ethnographic music. Its name recalls the origin of sound (acoustic) emission, i.e., it must be hit by some metallic components in order to produce acoustic effect. This instrument used to be performed only during the Holy Week before Easter, and it may have Byzantine origins. With his handler, the player retains the instrument and rapidly turns the carabattola right and left. In this way, the movement causes a clapper to hit in rapid sequence alternatively two metallic little circles, inserted in the wood. Thus, the clapper looks like a knocker and the acoustic effect appears.

The carabattola belongs to a series of musical instruments, all of them played during Holy Week before Easter in Mediterranean Europe. Other similar musical instruments are the Cembalo or Crotalo as Francesco Saverio Quadria, already reported in 1734 [13], normally made in oak or chestnut wood. Some other similar musical instruments, like Matracula, (Figure 1) are still used, although seldomly, in some parts of Italy such as the Sardinia region [14]. 


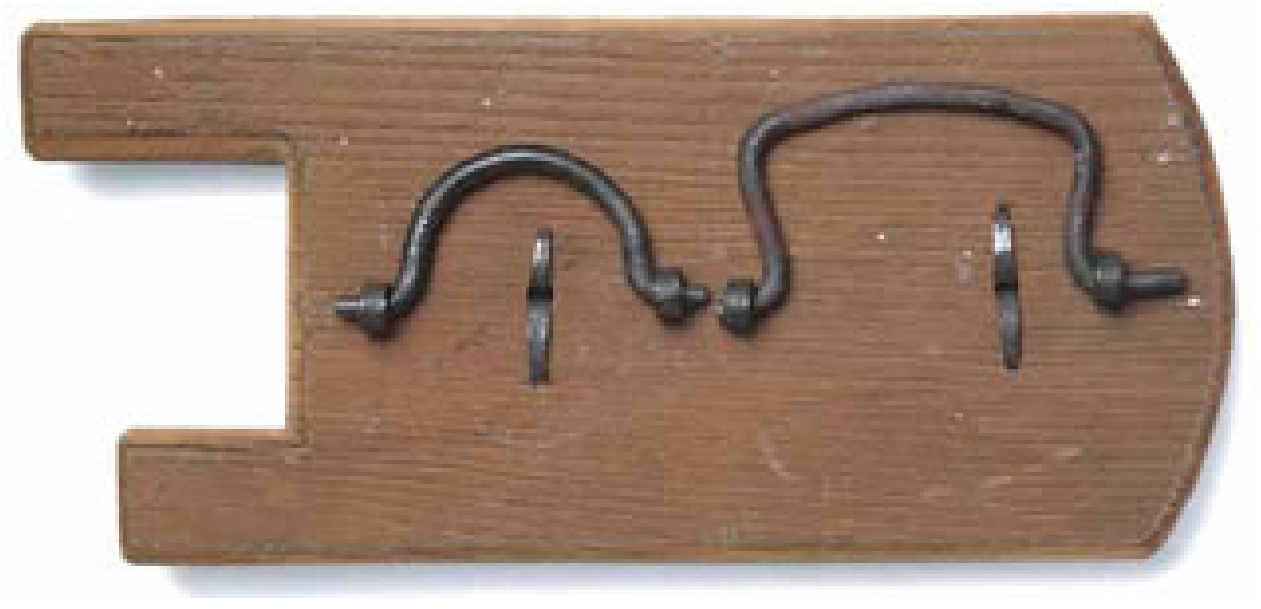

Figure 1. Matracula, a percussion instrument still in use in Sardinia (Italy).

The Carabattola, as well as the other similar musical instruments specifically realized for Holy Week, does not provide a specific pitched sound, but rather a particular acoustic effect, which increases the particular religious atmosphere, together with the chanting and the incense. These specific characteristics are the reasons for the decision to carry out vibrational and acoustic characterization of the wooden element (the "soundboard").

In this paper, an original example of Carabattola is analyzed. This instrument belongs to the Madonna del Carmine Church in Bagnacavallo, Ravenna, Northern Italy. It was locally called Scarabàtla and used to be played roughly until 1970. The chest (probably made by wood from fruit trees) is approximately $25 \mathrm{~cm}$ width, $50 \mathrm{~cm}$ length, $2.2 \mathrm{~cm}$ thickness, whilst the metallic (perhaps iron) circles are $2.0 \mathrm{~cm}$ width. It should be emphasized that this tool provides a background noise, partly comparable to the noise of a grater and not a real sound (as usually expected).

\section{Material and Methods}

\subsection{Acoustic Radiation}

The efficiency of acoustic radiation is a measure of the effectiveness of a vibrating surface in generating sound power. It could be defined by the relationship:

$$
\sigma=\frac{W}{\rho_{0} c S\left\langle\overline{v_{n}^{2}}\right\rangle}
$$

in which $W$ is the sound power radiated by a surface with area $S$, which could be obtained by integrating the far-field intensity over a hemispherical surface centered on the panel, and $\left\langle\overline{v_{n}^{2}}\right\rangle$ is the space-averaged value of the time-averaged normal distribution of velocity [15]. This parameter could be utilized for searching a link between movement (vibration) and acoustic generation (sound).

Various measuring techniques that are helpful for noise emission analysis could be acquired from this overall concept, ranging from frequent applications in noise emissions in machinery, to rare examples on musical instruments. In musical acoustics, previous investigations have been carried out on piano and harpsichord soundboards using different technique. Wogram used the parameter $F / v$, defining $\mathrm{F}$ as the excitation force and $\mathrm{v}$ as the resulting velocity at the point of excitation [16]. In his experiments, he found a maximum at a frequency that is close to or below $1 \mathrm{kHz}$ and that falls sharply below $100 \mathrm{~Hz}$ and above $1 \mathrm{kHz}$. He discovered that it falls typically by a factor of 10, as the frequency 
varies between 1 and $5 \mathrm{kHz}$. On the other hand, the "surface intensity method" was identified by Suzuki [10] and defined by the equation:

$$
I=\operatorname{Re}\left[p\left(\alpha / j \omega^{*}\right) / 2\right]
$$

where I represents the average intensity in time, perpendicular to the vibrating surface, measured in near field (about $30 \mathrm{~cm}$ from the radiating surface); $\omega$ is the angular frequency; Re and * are the real part and the complex conjugate of a complex number; $p$ and a are the pressure and the normal acceleration at the measuring point. Further, Giordano used the parameter $p / v$ where $p$ is the sound pressure measured in near field, and $\mathrm{v}$ is the velocity of the soundboard [11]. At around $1 \mathrm{kHz}$ in all sampled frequencies $p / v$ is larger and drops below a few hundred hertz and above $5 \mathrm{kHz}$.

It is essential to note that all these studies have one prevalent outcome: The frequencies of resonance have not coincided with those of acoustic emission, but they often have an adverse correlation.

\subsection{Intensity of Acoustic Radiation-IAR}

From the experiment described above, the Intensity of Acoustic Radiation (IAR) parameter was defined in 2005 as the space averaged amplitude of the cross-spectrum between the sound pressure generated from the vibration of the surface and the velocity of the surface vibration [17]:

$$
\operatorname{IAR}(\omega):=\langle P(\omega) * \mathrm{~V}(\omega)\rangle
$$

For the measurement, an omnidirectional microphone is required. According to Suzuki, the microphone must have been positioned in a specific point which corresponds to about one-quarter of the wavelength of the frequency corresponding to the earliest mode. If tympani are involved, this distance is approx. $25 \mathrm{~cm}$ over the membrane.

The measurements should be carried out in a slightly reverberating space where average radiation induced by early modes is easily achieved via the reverberation time. The acoustics chamber has no effect on the readings at higher frequencies. In addition, the measurements are enhanced by space-averaging of information carried out by shifting transductors through the instruments. It should be considered that other vibroacoustic parameters have been obtained starting from IAR, applied in very different field of acoustics, as building acoustics [18].

\subsection{I.A.R.: Previous Measurements}

When IAR was defined, in order to verify the robustness of the parameter, two distinct percussion instruments were used to measure IAR. These instruments were two kettledrums. The first was a 25-inch (approx. $65 \mathrm{~cm}$ ) kettledrum plexiglass Adam with a Remo mylar skin and central reinforcement tuned at about $166 \mathrm{~Hz}$ (corresponding to E), whilst the second one was a copper 25-inch Ludwig kettledrum, which was tuned to roughly $145 \mathrm{~Hz}$ (D-corresponding) with a mylar skin without a central reinforcer.

The tympani measurements were carried out in two different ways. A hammer with an accelerometer was used in the first case, whilst the hammer was replaced by a shaker in the second case. The microphone was in the same position in both cases. In order to verify the outcomes from previous researches, up to 15 modes were obtained and compared with literature. This allowed verifying the accuracy of the measurements. Figure 2 reports the results obtained in 2005 [17]. 


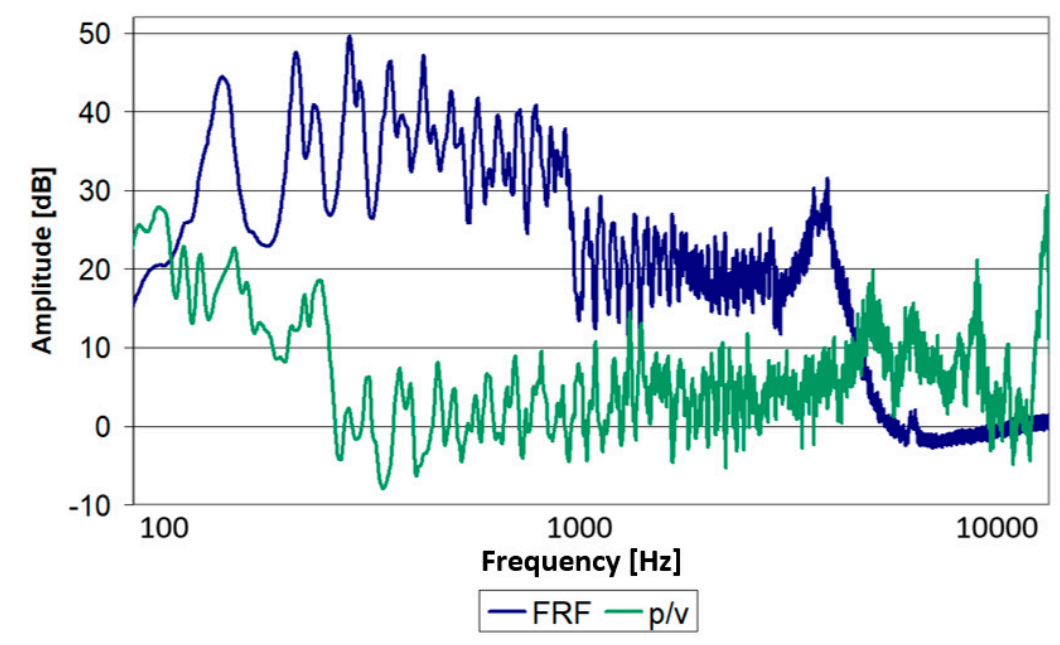

(a)

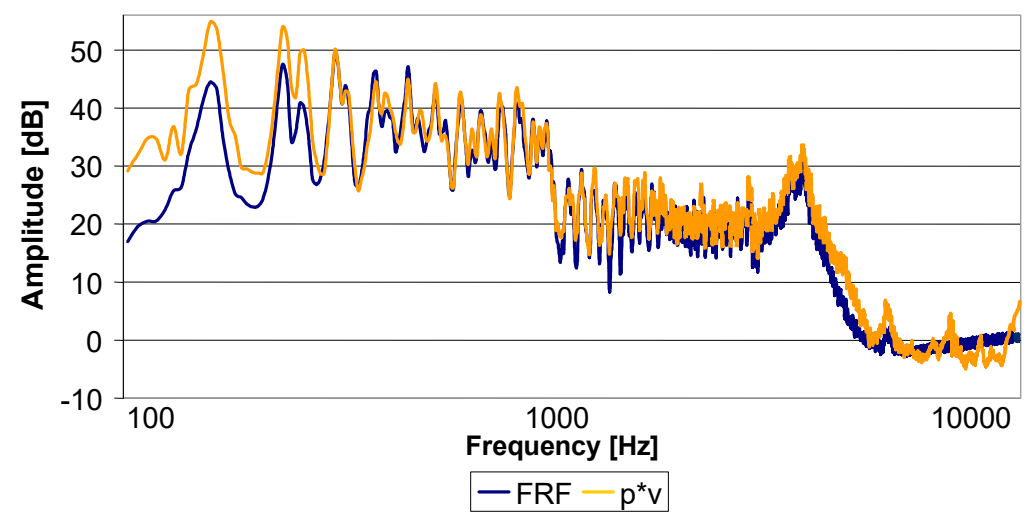

(b)

Figure 2. Tympani: (a) FRF compared with $p / v$; (b) FRF compared with IAR.

\section{Measurements on Carabattola}

The measurements on the carabattola were carried out in a similar way as the tympani. In one of two metal circles, the shaker excited the instrument, with the microphone positioned approximately 25 $\mathrm{cm}$ above. All measures were carried out in the same room of the tympani, with the same acoustic boundary conditions as described in the article [17].

Modal analyzes were also performed in this case. The measurements were conducted positioning the accelerometers on 24 positions and stored (Figure 3). In a further step, all the measurements were post processed in order to obtain FRF and modal patterns. The findings are quite different from the kettledrum, because the sound generation varies significantly from the tympani. The IAR is measured by the proportion $p / v$ and FRF as depicted in Figure 4 . Due to the increase in modal density at higher frequencies, the chart is restricted to $1 \mathrm{kHz}$. The modal patterns from 107 to $344 \mathrm{~Hz}$, measured in the instrument, are reported in Figure 5. 


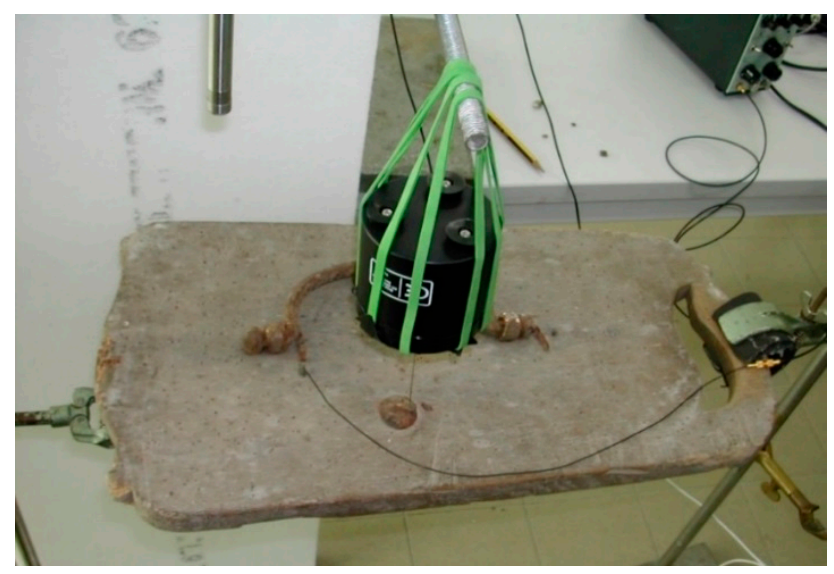

Figure 3. The measurements on the carabattola.

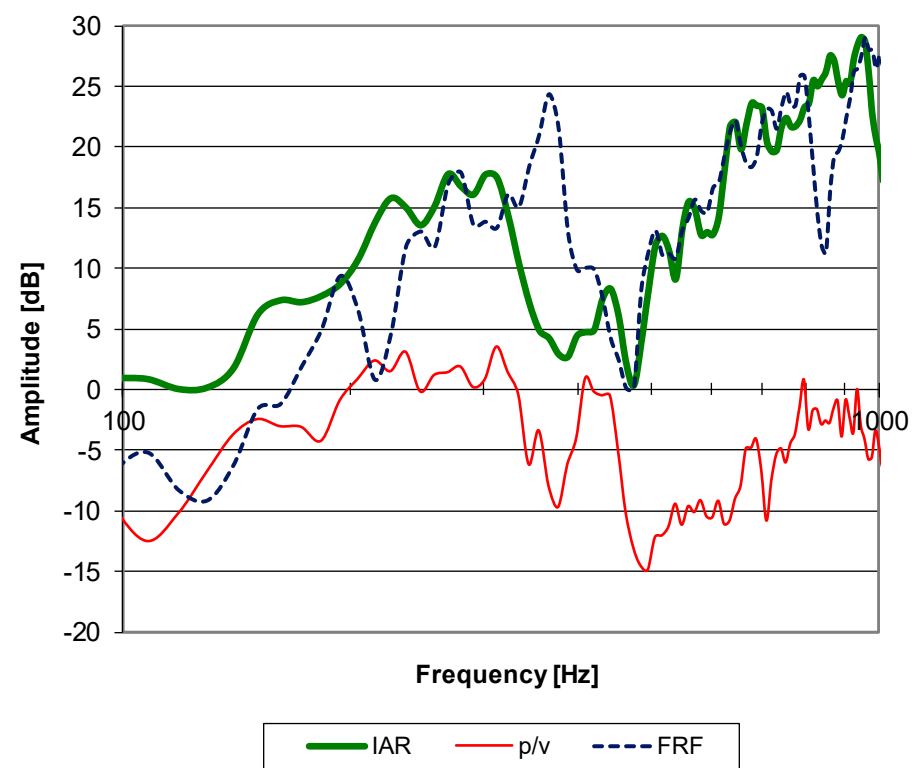

Figure 4. Carabattola: FRF compared with $p / v$ and IAR.

The match between IAR and FRF in carabattola is not as strong as in kettledrums. However, the negative correlation is not so obvious from FRF to $p / v$. The unique properties of sound emission of carabattola, which significantly differ from the tympanum, should explain this result.

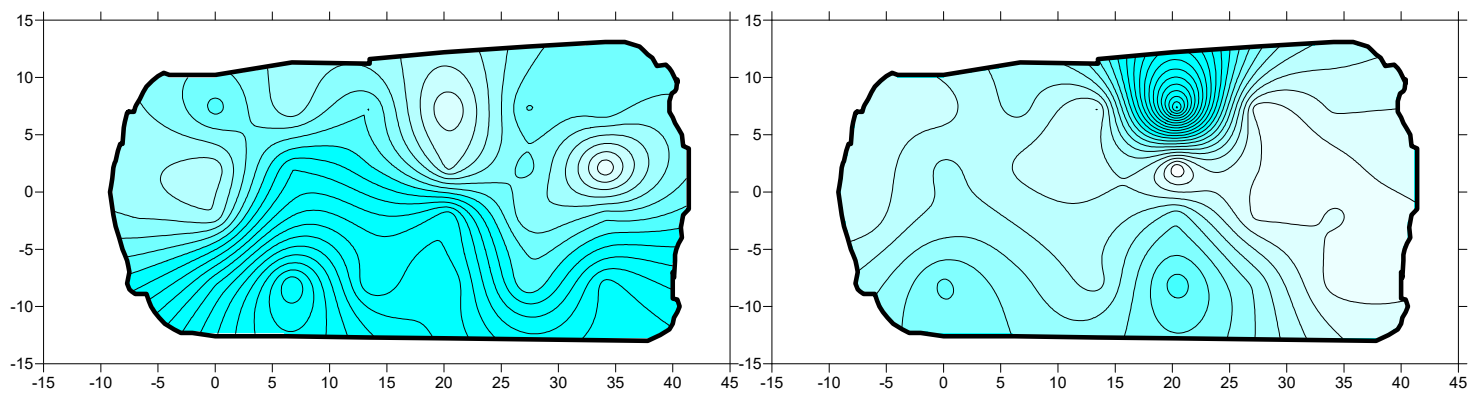

Figure 5. Cont. 

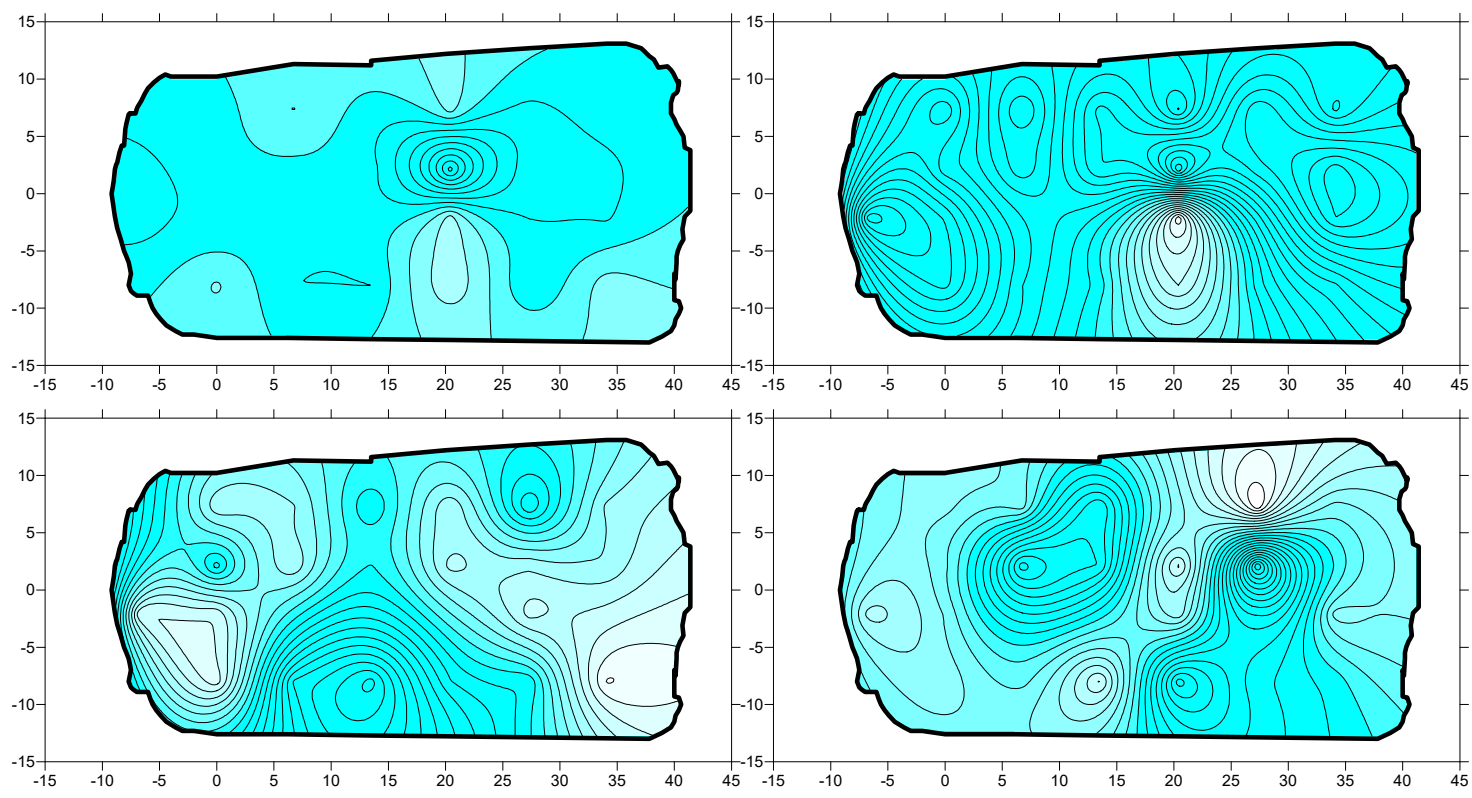

Figure 5. Modal patterns for the carabattola (from top left to bottom right): $107 \mathrm{~Hz}, 150 \mathrm{~Hz}, 193 \mathrm{~Hz}$, $247 \mathrm{~Hz}, 297 \mathrm{~Hz}, 322 \mathrm{~Hz}$.

The IAR measurements on the carabattola were also carried out together with modal analysis as previously made for the kettledrums. As mentioned earlier, the sound pressure $p$, as previously reported from Suzuki, Giordano and Tronchin, was evaluated in the near field at $25 \mathrm{~cm}$ from the instruments, maintaining the correct distance already proposed in early studies.

\section{Results}

\section{Analysis of Measured Data}

Comparing the results obtained on the carabattola with the results obtained on the kettledrums, the following points could be underlined.

In the case of the tympani, the negative correlations between FRF and IAR are very high, and at the same time, the two respective graphs are almost coincident. It should be noted that tympanum has a clearly defined sound pitch, mainly due to the membrane motion.

On the other hand, different results were carried out in the case of carabattola, as the mechanism of sound generation differs considerably from the tympanum. Figure 5 shows the first six modal carabattola patterns. It is important to note that the metallic plate where the knocker strikes the instrument affects the motion of the sound chest from the evaluation of these patterns. In view of the relationship between FRF and $p / v$, only partly a negative connection is noted, particularly at medium-low frequencies. In comparison, they are only partly linked, when comparing the two graphs FRF and IAR. The two charts seem like they are shifted of a small frequency range, even if they are much more linked than FRF and $p / v$.

The tympanum mainly produces sound from the membrane, which is a very straightforward perception of the pitch caused by the membrane vibration. On the other hand, in the carabattola the chest and metal knocker produce a sound that is not perceived as a particular pitch.

In the case of tympani, IAR, FRF and $p / v$ are highly correlated each other, as almost all sound generation comes from the membrane (where the sound velocity $\mathrm{v}$ is measured), whilst the correlation in carabattolas is not as obvious as the sound generation comes from wood alone. 


\section{Discussion}

Two types of percussion instruments, two tympani and one carabattola, were carried out with acoustic radiation measurements and modal analysis, as proposed by many authors in previous articles. In both cases, the instruments were excited by means of a shaker, connected with a thin metallic bar. In a previous paper, the shaker resulted better than the head impedance hummer, as the resonance of the bar that connects the shaker with the instruments was discovered at approximately $3 \mathrm{kHz}$.

The mappings of individual vibration modes were very clear for all the instruments, and in the case of tympani, the frequency ratios were approximately consistent with the theory. For circular and mixed vibration modes, a high degree of correspondence was obtained, while the diametric modes produced slightly less frequencies than the theoretical ones. Due to the typical noise generated in the carabattola, modal pattern mapping was not so evident like in the tympanum.

The tympanum generates its sound mainly from the membrane and has a very little thickness if compared with its surface and could be considered bi-dimensional. The membrane is elastically deformable, and its vibration is only slightly damped. Conversely, the carabattola is made of a wooden body having a relevant thickness of $2.2 \mathrm{~cm}$, not elastically deformable and cannot be considered bi-dimensional. The body is not elastically deformable, and its vibration is strongly damped.

Acoustic radiation has been evaluated in two different ways in all musical instruments. The first step was to calculate the complex ratio $(p / v)$ between sound pressure and vibration velocity of the principal vibration components of the instrument. This is the method used by Giordano.

In the second step, an amplitude of a space-averaged of cross spectrum $(p \cdot v)$ between sound pressure and the vibrational speed of the membranes or chest was calculated at a fixed point $25 \mathrm{~cm}$ over the instruments. This is the parameter known as Intensity of Acoustic Radiation (IAR).

The IAR thus led to a parameter that was able to correctly associate the vibration of plate or membranes with the sound production. This relationship is remarkable for tympani, while the relationship is not so strong for carabattola. In the first situation, IAR and FRF are very correlated and only partly linked in the second situation. We have not considered the effect of the metal knocker, because our focus was in the vibroacoustic behavior of the soundboard.

Considering the modal patterns obtained from modal analysis and reported in Figure 5, it is noticeable that there are no clear modal patterns obtained from the analysis. This result is coherent with the sound behavior of the musical instrument, which is not characterized by a specific pitch but rather produces a strong peculiar noise which recalls a grater. Since there is not any specific pitch in the sound emission, there are no frequencies that emerged from the music spectrum. Moreover, the thickness of the soundboard, which is considerably higher than that of other wooden musical instruments or even plates, causes an early damping of the sound (or noise) emission that is compensated by the quick movement of the metallic handle, which hits the soundboard with a very high frequency.

In other words, the sound analysis carried out on the carabattola does not give evidence of any specific frequency but rather looks partially similar to a flatten spectrum without any decay, similarly to percussion instruments like a wooden scraper. Consequently, the modal patterns which resulted from the analysis resulted having an irregular shape, which is completely different from the membrane of the tympanum.

\section{Conclusions}

The carabattola is an almost unknown idiophone musical instrument that used to be played only during Holy Week in Mediterranean Europe. Few examples of this musical instrument are actually conserved in Italy, and no acoustic analyses are available for it. This paper attempted to describe its vibroacoustic behavior by means of some specific acoustic measurements: modal analysis and intensity of acoustic radiation. These methods have been already utilized in other musical instruments, as the kettledrum. 
In the carabattola, comparing the FRF graph and $p / v$, it can be noted how frequencies of resonance often differ from those of acoustic emission. When IAR is applied, resonance frequencies completely match those of noise emissions, and the forms of the two graphs are comparable.

In the case of tympani, this was particularly evident: The IAR parameter was well related to the response function of frequencies, and therefore, it resulted preferable to $p / v$. IAR is a parameter between acoustic intensity and acoustic radiation, so it is suitable to measure the sound generating characteristics of musical instruments with vibrating soundboards (i.e., FRF). This parameter could also be used to describe and identify the directivity of musical instruments, essential both for architectural acoustics and for procedures of auralization.

In the case of the kettledrum, the links between FRF, IAR and modal analysis were very high, because of the clear subjective pitch of the M.I. In the case of the carabattola, where no pitch is detected, the correlations between FRF, IAR and modal patterns resulted very low. These results underline the link existing between IAR, modal patterns and pitch of the vibrating surface: Where no pitch is detected, low correlations occur between IAR and FRF, and modal analysis becomes less important.

The results obtained in the carabattola showed that modal patterns were not clearly identified as in the violin or kettledrum, and its sound emission was characterized by an almost uniformed frequency distribution, which typically characterizes idiophones.

Author Contributions: L.T. provided the funding, made the measurements and the results. M.M. contributed to the writing of the paper. V.V. finalized the paper including its formatting. All authors have read and agreed to the published version of the manuscript.

Funding: This research was funded by Italian Government in the framework of PRIN 2015. within the project "Research for SEAP: a platform for municipalities taking part in the Covenant of Mayors".

Acknowledgments: The Authors wish to thank Elisa Ferri for her precious help during the measurements and post processing.

Conflicts of Interest: The authors declare no conflict of interest.

\section{References}

1. Woodhouse, J. The acoustics of the violin: A review. Rep. Prog. Phys. 2014, 77, 115901. [CrossRef] [PubMed]

2. Askenfelt, A.; Jansson, E.V. From touch to string vibrations. I: Timing in the grand piano action. J. Acoust. Soc. Am. 1990, 88, 52-63. [CrossRef]

3. Askenfelt, A.; Jansson, E.V. From touch to string vibrations. II: The motion of the key and hammer. J. Acoust. Soc. Am. 1991, 90, 2383-2393. [CrossRef]

4. Farina, A.; Tronchin, L. On the "virtual" reconstruction of sound quality of trumpets. Acust. United Acta Acust. 2000, 86, 737-745.

5. Tronchin, L.; Manfren, M.; Vodola, V. Sound characterization through IAR measurement: A study of Persian Musical Instruments. Appl. Sci. 2020, 10, 633.

6. Tronchin, L. The Emulation of Nonlinear Time-Invariant Audio Systems with Memory by Means of Volterra Series. J. Audio Eng. Soc. 2012, 60, 984-996.

7. Farina, A.; Langhoff, A.; Tronchin, L. Acoustic characterisation of "virtual" musical instruments: Using MLS technique on ancient violins. J. New Music Res. 1998, 27, 359-379. [CrossRef]

8. Tronchin, L.; Coli, V.L. Further investigations in the emulation of nonlinear systems with Volterra series. J. Audio Eng. Soc. 2015, 63, 671-683. [CrossRef]

9. Carini, A.; Orcioni, S.; Terenzi, A.; Cecchi, S. Nonlinear system identification using Wiener basis functions and multiple-variance perfect sequences. Signal Process. 2019, 160, 137-149. [CrossRef]

10. Suzuki, H. Vibration and sound radiation of a piano soundboard. J. Acoust. Soc. Am. 1986, 80, $1573-1582$. [CrossRef]

11. Giordano, N. Sound production by a vibrating piano soundboard: Experiment. J. Acoust. Soc. Am. 1998, 104, 1648-1653. [CrossRef]

12. Christian, R.S.; Davies, R.E.; Tubis, A.; Anderson, C.A.; Mills, R.I.; Rossing, T.D. Effects of air loading on tympani membrane vibrations. J. Acoust. Soc. Am. 1984, 76, 1336-1345. [CrossRef] 
13. Quadrio, F.S. Della storia e della ragione d'ogni poesia volumi quattro di Francesco Saverio Quadrio. In Milano, nelle stampe di Francesco Agnelli; nelle stampe di Francesco Agnelli: Milano, Italy, 1741.

14. Spanu, G.N. SONOS-strumenti della musica popolare sarda Ilisso Editore. 1994. Available online: https: //www.libreriauniversitaria.it/sonos-strumenti-musica-popolare-sarda/libro/9788885098305 (accessed on 15 January 2020). (In Italian)

15. Fahy, F.J. Sound Intensity; Elsevier: London, UK, 1989.

16. Wogram, K. Akustische Untersuchungen an Klavieren, in Der Piano -und Flÿgelbau Verlag Das Musikinstrument, Frankfurt; English version, Acoustical Research on Pianos: Vibrational Characteristics of the Soundboard. Das Musikinstrument 1984, 24, 380-404.

17. Tronchin, L. Modal analysis and intensity of acoustic radiation of the kettledrums. J. Acoust. Soc. Am. 2005, 117, 926-933. [CrossRef] [PubMed]

18. Piana, E.A.; Petrogalli, C.; Paderno, D.; Carlsson, U. Application of the wave propagation approach to sandwich structures: Vibro-acoustic properties of aluminum honeycomb materials. Appl. Sci. 2018, 8, 45. [CrossRef]

(C) 2020 by the authors. Licensee MDPI, Basel, Switzerland. This article is an open access article distributed under the terms and conditions of the Creative Commons Attribution (CC BY) license (http://creativecommons.org/licenses/by/4.0/). 